\title{
Chapter 16 \\ Fuzzy Numbers Applied to a Heat Furnace Control
}

\author{
Wojciech T. Dobrosielski, Jacek M. Czerniak, Hubert Zarzycki \\ and Janusz Szczepański
}

\begin{abstract}
This chapter presents a trend phenomenon and application of the fuzzy controller for Ordered Fuzzy Numbers (OFNs). The authors propose to use a trend in a combustion process for a simplified model of a solid fuel fired furnace. Better control over the process translates into reduced $\mathrm{CO}_{2}$ emission as well as optimal use of the furnace. When carrying out the fuzzy observation of the efficiency of the furnace, the authors apply the OFN notation by connecting the trend of furnace temperature changes with the order appropriate for this notation. Thanks to this approach it is possible to enhance information without the additional need to multiply the transmitted data. It is particularly effective in the multidimensional fuzzy observation when monitoring not only the condition of the temperature in the furnace but also the ambient temperature and the temperatures in several rooms of the heated building. The chapter is a continuation of a series of papers published by the authors on multidimensional fuzzy observation using OFN notation. A controller in the conventional fuzzy logic approach is also presented in the chapter. The controller was built using jFuzzyLogic software. The fact that there are more and more OFN applications seems to be a good predictor of the development of this generalization, an example of which is the problem analyzed in this chapter.
\end{abstract}

W.T. Dobrosielski $(\varangle) \cdot$ J.M. Czerniak

Kazimierz Wielki University, Institute of Technology, ul. Chodkiewicza 30,

85-064 Bydgoszcz, Poland

e-mail:wdobrosielski@ukw.edu.pl

J.M. Czerniak

e-mail: jczerniak@ukw.edu.pl

H. Zarzycki

University of Information Technology and Management Copernicus,

ul. Inowrocławska 56, 53-648 Wrocław, Poland

e-mail: hzarzycki@yahoo.com

\section{J. Szczepański}

Institute of Fundamental Technological Research, Polish Academy of Sciences,

ul. Pawinskiego 5B, 02-106 Warsaw, Poland

e-mail: jszczepa@ippt.gov.pl 
Keywords Adaptive systems • Ordered Fuzzy Number - Inference - Defuzzification $\cdot$ Fuzzy hardware $\cdot$ Fuzzy software $\cdot$ Fuzzy control

\subsection{Introduction}

The development of civilization is associated with an increase in energy demand. In modern times a variety of technologies is used for generating electricity. Nuclear power is one of these technologies supposed to solve the problem of energy shortage. Assumptions proved to be wrong, as for the atom and other fuels [59]. People should look for alternatives. An important factor in obtaining energy is the amount of carbon dioxide produced, which contributes to climate change. It is assumed that it is impossible fully to eliminate fuel combustion resulting in carbon dioxide. Reducing emissions of $\mathrm{CO}_{2}$ in industrial processes is the optimal solution with regard to the interests of individual countries. The main activities that can reduce $\mathrm{CO}_{2}$ emissions include reduction of energy consumption, but significant reduction seems unlikely. There is also the possibility of increasing the use of carbon-free renewable energy sources such as solar, wind, hydropower, and geothermal resources [59]. Increasing the efficiency of energy conversion to useful energy, as the last element affecting the reduction of $\mathrm{CO}_{2}$ is a proposal the authors intend to develop based on the capabilities of artificial intelligence, in particular based on fuzzy logic. According to the authors the possibility of such process control, which improves combustion efficiency, whether in domestic or industrial stoves, and will reduce emissions of harmful compounds, is remarkable. The combustion process is a chemical reaction of oxidation that takes place between fuel and oxygen. The result is an exotherm and the formation of flue gases. The general process of burning coal is shown in a simplified chemical formula below.

$$
\mathrm{C}+\mathrm{O}_{2} \rightarrow \mathrm{CO}_{2}+\text { released heat }
$$

The highest combustion temperature is obtained using the least amount of oxygen at which there is no free oxygen in the exhaust gas. In other words, the idea is that all the ingredients are oxidized to $\mathrm{CO}_{2}, \mathrm{H}_{2} \mathrm{O}, \mathrm{SO}_{2}$. Oxygen-enriched combustion lowers the temperature giving a cooling effect. Combustion with oxygen depletion also reduces the temperature, thereby contributing to a partial combustion of the fuel. In contrast, a process that is desirable according to the literature [37] is called a stoichiometric process. The mixture in this embodiment is burned completely and exhaust gases contain neither fuel nor oxygen. Ensuring proper combustion, which depends on many factors (e.g., the calorific value of the fuel, the construction and function of the stove), may be a difficult control problem. Redefining Eq. 16.1 as the basic equation describing the relationship between the combustion components is represented by Eq. 16.2. 


$$
\frac{100}{\mathrm{CO}_{2}} *\left[\mathrm{CO}_{2}\right]+\frac{100}{21} *\left[\mathrm{O}_{2}\right]+\left[\frac{100}{\mathrm{CO}_{2}}-\frac{79}{21}\right] *[\mathrm{CO}]=100
$$

Equally Eq. 16.2 can be represented as an Ostwald graph [63], where this relationship for lignite is shown in Fig. 16.1. Using this graph allows regulation of the combustion process. When the measuring point is on the line between the axes of the carbon dioxide and oxygen, the carbon monoxide content is $0 \%$. If a point is above the line it means that an error has occurred. The situation when the point is below the line in the combustion means that there is incomplete combustion and in the exhaust gas there is carbon monoxide in addition to carbon dioxide. Accordingly, Fig. 16.1 shows a coefficient $\lambda$, which is expressed as $\phi$. The general model of the stove on solid fuel for the purpose of this work is shown below Fig. 16.2. The diagram shows the essential elements, which are discussed later. Imprecision of factor borders accompanying the combustion process has contributed to the use of fuzzy logic. This is confirmed by numerous publications, where examples of the work are [30, 56]; especially important in the context of this article there is the study by [56]. Depicting a fuzzy system of monitoring carbon dioxide in the combustion process is a Takagi-Sugeno inference model [62]. Combining fuzzy logic with any process or phenomenon, in which we use a linguistic description of reality is a broad field of research. Lotfi A. Zadeh can be considered the creator of fuzzy logic. In 1965 this American professor published an article "Fuzzy Sets" [65] in the journal, Information and Control, defining the notion of a fuzzy set, where inaccurate information is described by values from the interval $(0,1)$.

Fig. 16.1 Ostwald diagram for lignite based on [63]

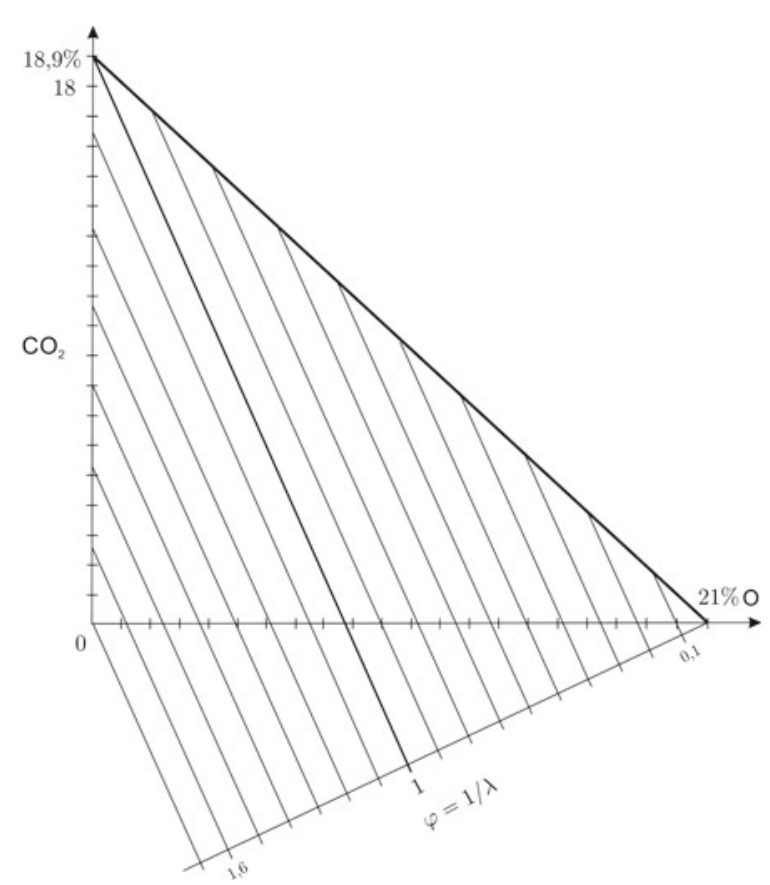


Fig. 16.2 Indicative graph of a solid fuel stove

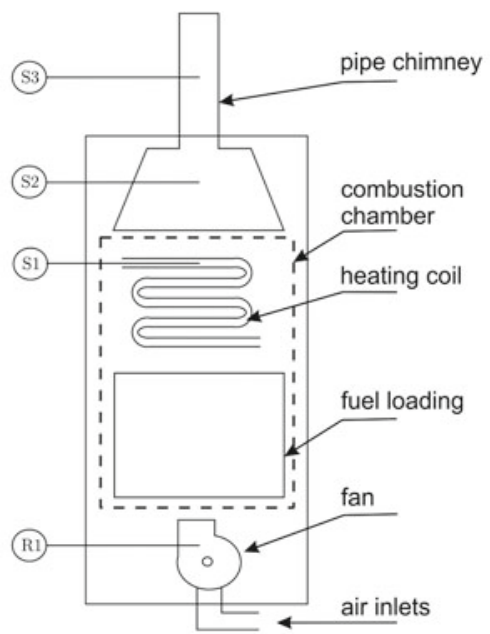

S1, S2, S3 - sensors, R1 - fan speed regulator

It can also be said that the precursor of Zadeh's achievements is the Polish mathematician Jan Łukasiewicz, who in the 1950s published an article on three-valued logic [39]. The formulation of the foundations of fuzzy logic and the involvement of the scientific community have contributed to the creation of many theories expanding this subject. One of them is the representation of the L-R fuzzy set. The authors Dubois and Prade in [16] introduced the limited membership functions of the two functions of the shape of $\mathrm{L}$ and $\mathrm{R}$. The linear shape function operations on the numbers L-R gave the partial opportunity to reproduce results for the triangular membership function. In the case of multiplication, the resulting number of L-R led to enlarging the range introducing imprecise intervals.

We now depart from the genealogy of fuzzy logic development to address again the original problem, the process of controlling the stove on solid fuel. The authors' inspiration to write this chapter was that the combustion process can benefit from the trend. The trend is visible when the stove water temperature rises to a specified level; the upward trend may be shaken by the excess of supplied oxidant. Oxygen contained in the exhaust gases and the quantitative composition result in the combustion process. For example, reaching the required water temperature during firing of the stove is associated with the fan modulation to $80-100 \%$ of power and leads to too lean a mixture. The effect of an excessive amount of oxidant is the cooling of the heat transfer medium. The water temperature decreases instead of increasing. Temporary and long-term ups and downs translate into the trend of the process [7-9, 11-15]. Another example might be the work of [6, 21, 38, 41-43, 57, 58], in which the authors use the trend as a direction in the problems of management accounting and in determining the internal rate of return (IRR) for investment or other issues of recognition attack computer networks described in [1-4], and also when talking about engineering solutions [7-14, 44-49, 60, 61, 68]. The opportunity to study 
the behavior of phenomena expressed as a trend in fuzzy logic is possible thanks to directing as proposed by the team of Professor W. Kosiński. The authors' proposal [34] concerns the redefinition of fuzzy numbers as directed fuzzy numbers OFNs (Ordered Fuzzy Numbers), where the main advantage is the ability to solve a linear equation 16.3 .

$$
A+B=C
$$

where A, B are any Ordered Fuzzy Numbers for which the following equality should occur.

$$
A=C-B
$$

In the case of using numbers proposed by Dubois and Prade, Eq. 16.4 will be false. This is because of fuzzy number imprecision expansion. Using OFNs enables easyto-perform arithmetic operations on them and on the real numbers. In addition, one can use direction as the trend of the process.

\subsection{Selected Definitions}

\subsubsection{The Essence of Ordered Fuzzy Numbers}

The basic concept of Ordered Fuzzy Numbers has been described in the introduction. In this chapter a deeper concept of OFNs is presented. This is essential for understanding the solutions used in the chapter. The authors of OFN are the team of Professor W. Kosiński, P. Prokopowicz, and D. Ślęzak [31-36, 51-55]. The problem of increasing imprecision with the increasing number of performed operations and the lack of solutions to Eqs. 16.3 and 16.4 in fuzzy logic has been noted by the authors. Redefining classic fuzzy sets, which is according to Zadeh to postulate an ordered pair, expanded the definition of an ordered pair of functions. The number is defined there as follows.

Definition 1 Ordered Fuzzy Number A is an ordered pair of functions

$$
A=\left(x_{\text {up }}, x_{\text {down }}\right)
$$

where $x_{\text {up }}, x_{\text {down }}:[0,1] \rightarrow R$ are continuous functions.

These functions are called, respectively, the up- and down-parts. These two parts are connected via a constant function equal to 1 on the interval. The direction of a fuzzy number is called the orientation: the up-part of the OFN is the beginning and the down- part is the end of this number. A graphic interpretation of Ordered Fuzzy Numbers is presented on the left side of Fig. 16.3, and the right side is the directed number with a reference to classical fuzzy numbers. 

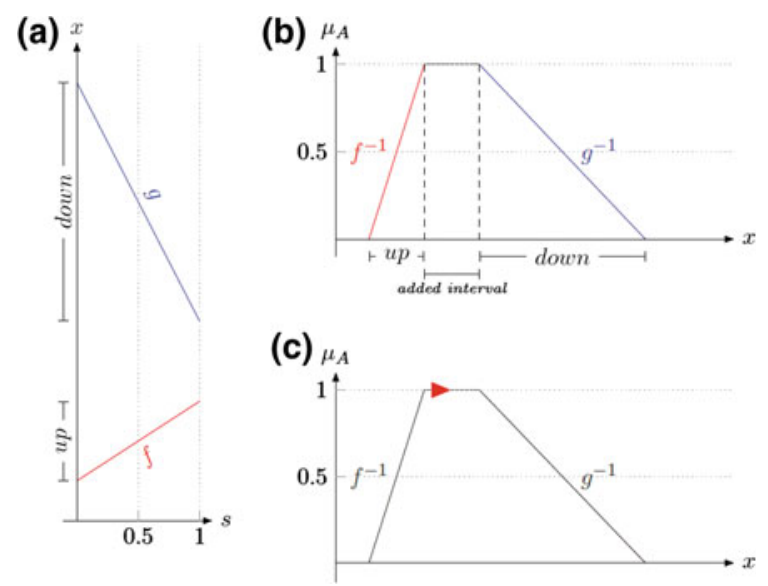

Fig. 16.3 a OFN example, b OFN presented with a reference to classical fuzzy number, and c simplified mark denoting the order of inverted functions

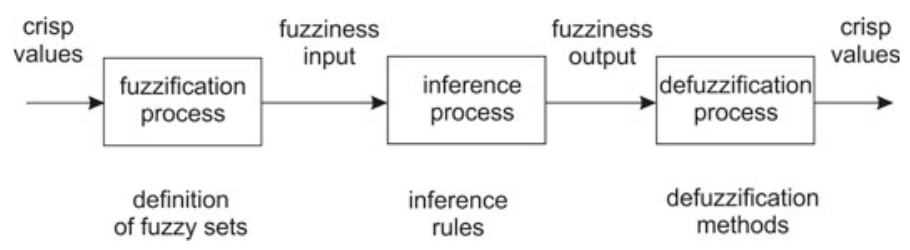

Fig. 16.4 Schematic diagram of the fuzzy controller

\subsubsection{Fuzzy Controller}

The formulation of fuzzy controller mathematical foundations can be found in the literature [28, 29, 40, 64, 66, 67]. These subject-related concepts using linguistic variables assume the role of describing input and output states, which we intend to express and assess a linguistic description. Linguistic value directly affects the verbal assessment of the linguistic object. For example, the linguistic variable "voltage" takes the linguistic values of "small, medium, and high." The process of fuzzy control is shown in Fig. 16.4, which includes operations such as fuzzification, inference, and defuzzification. The fuzzification operation is carried out in the first stage. It is associated with the calculation of the degree of membership to particular fuzzy sets. In the inference stage, based on the input degree of membership we calculate the resulting membership function. An important conclusion is that the expected outcome is to define the system resulting in the membership function. Both fuzzification and inference operations contain a number of specific elements.

The closing operation of the system is the defuzzification block. It is characterized in that the output is a specific (not fuzzy) value. This value is the product of the method operating on the resulting membership function and enables the activation of the actuator in the desired manner. 
Defuzzification is therefore a function that assigns a crisp value to a fuzzy number. There are several well-described fuzzification functionals, which are utilized in many arithmetics used in fuzzy logic, including the following.

\section{FOM: First of Maxima}

The FOM method is a method of selecting the smallest element of the set $A$ kernel, where the fuzzification value is described in Eq. 16.6.

$$
\operatorname{FOM}(A)=\min \operatorname{core}(A)
$$

\section{LOM: Last of Maxima}

Accordingly selecting the highest value of the set $A$ kernel, we use the LOM method; the formula is as below:

$$
\operatorname{LOM}(A)=\max \operatorname{core}(A)
$$

\section{MOM: Mean of Maxima}

Equation 16.8 shows the use of LOM and FOM as a method for which the fuzzification value includes the minimum and maximum elements of the $A$ fuzzy set kernel. This value is the average of these two methods.

$$
\operatorname{MOM}(A)=\frac{\min \operatorname{core}(A)+\max \operatorname{core}(A)}{2}
$$

\section{RCOM: Random Choice of Maxima}

The method is also called kernel fuzzification because the fuzzification value is always contained in the kernel of a fuzzy set. The fuzzification value of this method is a random element $x \in \operatorname{cor} e(A)$ calculated as the probability of:

$$
\operatorname{RCOM}(A)=P(x)=\frac{\lambda(x)}{\lambda(\operatorname{core}(A))}
$$

where $\lambda$ is the Lebesgue measure in universe $X$.

\section{MOS: Mean of Support}

This method shows the fuzzification value is the average of the $A$ number medium.

$$
\operatorname{MOM}(A)=\frac{\operatorname{supp}(A)}{2}
$$

\section{COG: Center of Gravity}

This is the most common method in cases where it is important to determine the center of gravity of the considered system. In the fuzzification process of an $A$ fuzzy number, the COG method is expressed as Eq. 16.11. 


$$
\operatorname{COG}(A)=\frac{\int_{a}^{b} x \mu_{A}(x) \mathrm{d} x}{\int_{a}^{b} \mu_{A}(x) \mathrm{d} x}
$$

\section{BADD: Basic Defuzzification Distribution}

The method of fuzzification was proposed in [20] as an extension of the COG and MOM methods. The fuzzification value of the fuzzy set $A$ is obtained as

$$
B A D D(A)=\frac{\int_{a}^{b} x \mu_{A}^{\gamma}(x) \mathrm{d} x}{\int_{a}^{b} \mu_{A}^{\gamma}(x) \mathrm{d} x}
$$

It is also worth paying attention to the methods dedicated to OFN arithmetic, which are inherently sensitive to directing. These include: Fuzzification method called the Golden Ratio (GR): It was established as a result of implementation of an ancient division used in Greek architecture. The GR value for the fuzzy number $A$ is given by the equation:

$$
G R(A)=\left\{\begin{array}{l}
\min (\operatorname{supp}(A))+\frac{|\operatorname{supp}(A)|}{\Phi}, \text { if order }(A) \text { is positive } \\
\max (\operatorname{supp}(A))-\frac{|\sup (A)|}{\Phi}, \text { if order }(A) \text { is negative }
\end{array}\right.
$$

where $G R$ is the defuzzification operator, $\operatorname{supp}(A)$ is support for fuzzy set $A$ in universe $X$, and $\Phi=1,618033998875 \ldots$.

Mandala factor (MF) method: This is the calculation of the $\Re$ value using the Mandala factor $\Psi_{A}$ sum of function integrals of a rising edge, falling edge, and a core set. The resulting value is scaled from the center of the coordinate system by adding it to the beginning value of a fuzzy number support set. When fuzzification is carried out in OFN arithmetic, in the case of a positive directing procedure described as above, and in the case of negative directing a calculated negative value should be subtracted from the first coordinate of an OFN number being the OFN number support right edge.

$$
\operatorname{MF}(A)=\left\{\begin{array}{l}
c+r, \text { if order }(A) \text { is positive } \\
c-r, \text { if order }(A) \text { is negative }
\end{array}\right.
$$

where

$$
\begin{array}{r}
r=\frac{1}{d-c} \int_{c}^{d} x d x-\frac{c}{d-c} \int_{c}^{d} d x+\frac{f}{f-e} \int_{f}^{e} d x \\
-\frac{1}{f-e} \int_{e}^{f} x d x+\int_{d}^{e} d x
\end{array}
$$

Modified center of gravity: This is the result of modifications introduced by Kosiński and Bednarek [5] in the classical COG method in order to adapt it to work in OFN arithmetic. 
(a)

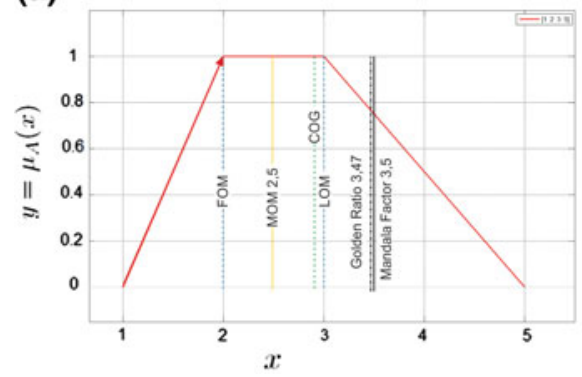

(b)

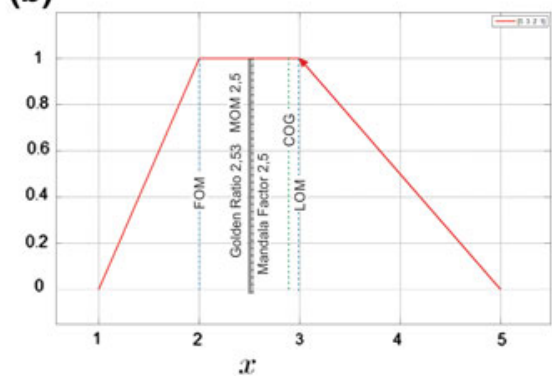

Fig. 16.5 Graphical representation of selected fuzzification functionals for the same OFN number directed a positively and $\mathbf{b}$ negatively

$$
\begin{aligned}
& \Psi_{C O G}(\zeta, f, g)=\frac{\int_{0}^{1}(\zeta g(s)+(1-\zeta) f(s))|g(s)-f(s)| d s}{\int_{0}^{1}|g(s)-f(s)| d s}, \\
& \text { if } f(s) \neq g(s) \\
& \qquad \Psi_{C O G}(\zeta, f, g)=\frac{\int_{0}^{1} f(s) d s}{\int_{0}^{1} d s}, \text { if } f(s)=g(s)
\end{aligned}
$$

Figure 16.5a, $b$ are drawings showing different fuzzification results for the same OFN directed positively in Fig. 16.5a, and directed negatively in Fig. 16.5b. Calculations were performed using selected fuzzification functionals.

\subsubsection{Control of the Stove on Solid Fuel}

Referring to the example of the stove fuzzy controller, a diagram of such a system is shown in Fig. 16.6, where $S 1$ is a heater temperature sensor, $S 2$ a temperature or exhaust lambda sensor, and $S 3$ an optional gas analyzer. The stove uses an air blower. In this example it is called a blower regulator $R 1$. The basic concept of the work of the control system is as follows. Input variables that were selected are:

- Water coil temperature: $T w$

- Stove flue gas temperature: $T s$

- Lambda probe: $\lambda$

- Increase of temperature: $\Delta T t$ 
Output variables are:

- Speed of the blower

There are additionally provided auxiliary variables for the solution, $T z$, set temperature, which says what temperature the user prefers, or what temperature is optimal for a particular stove. For example, the OFN describing set temperature $T Z 1=[56,56,56,56]$ informs the controller to "hold temperature of 56" For the number $T Z 2=[56,58,60,72]$ the directing means, "I prefer temperature 72, but may be no less than 56." For the reverse OFN number there is the statement, "I prefer 56 , but nothing will happen if it is $72 . "$

Linguistic variables selected for this problem depend on the concept of the control operation. For example, the observed coil temperature is lower than the preset temperature. The stove driver starts the blower and observes the flue gas temperature or $\lambda$ probe. In both cases, the sensors provide information about the progress of the operation of the stove. Based on this information, the controller determines the amount of air sent by the blower.

\subsection{Classic Fuzzy Controller}

The controller in the classic use of fuzzy logic was done using jFuzzyLogic. The technical details are available at http://jfuzzylogic.sourceforge.net. The input linguistic variables as described in the previous chapter are provided as fuzzy sets. Accordingly, we have Figs. 16.7 and 16.8.

Fig. 16.6 Fuzzy logic controller dedicated to solid fuel stove

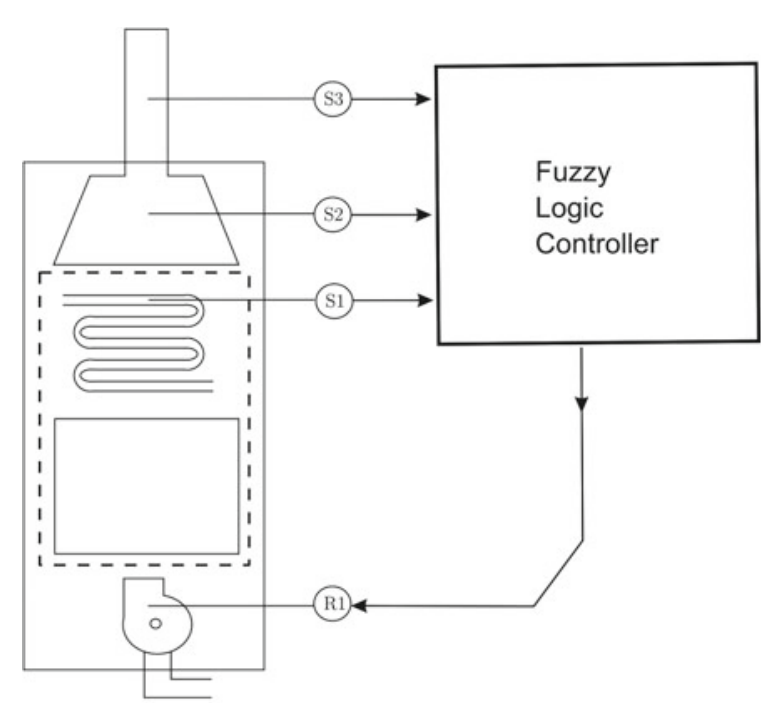

S1, S2, S3 - sensors, R1 - fan speed regulator 


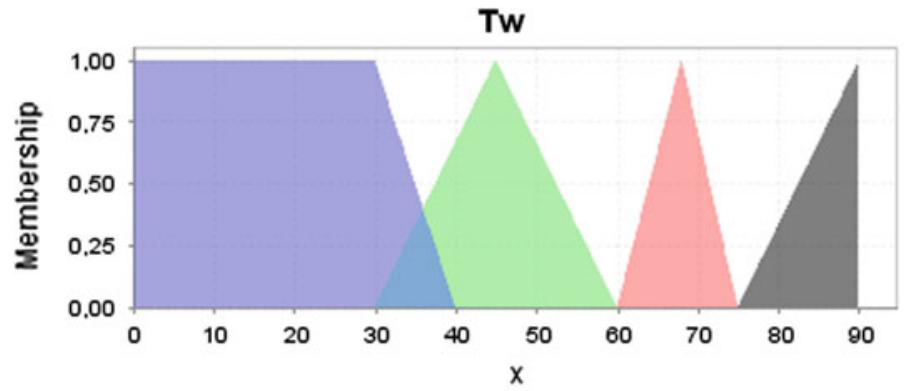

- very_high $\mathbf{a}$ high $\mathbf{a}$ low $=$ average

Fig. 16.7 Input variable 'coil temperature' and its fuzzy set

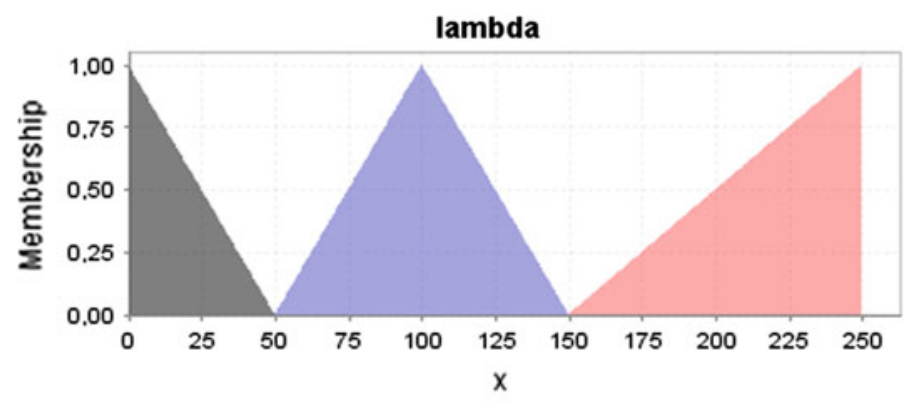

a rich a poor a optimal

Fig. 16.8 Input variable 'lambda' and its fuzzy set

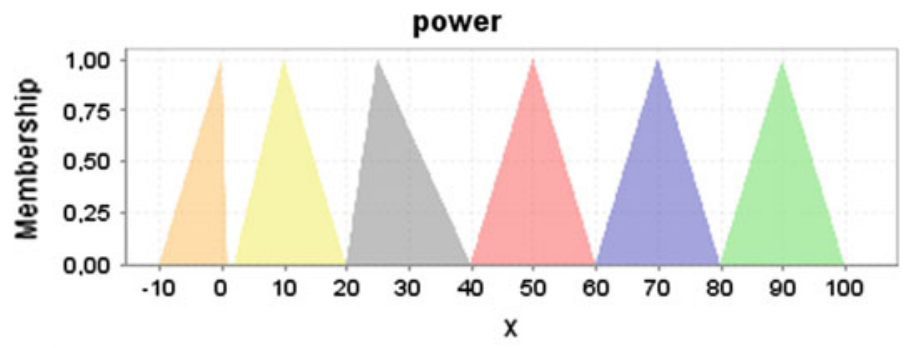

a power:0,00 (CenterOfGravity) $\approx$ power_40 $\approx$ power_60 $\approx$ power_80 a power_100 power_20 a power_0

Fig. 16.9 Output variable 'power' and its fuzzy set 
The output variable in the example is the power of the blower regulator. Figure 16.9 shows a membership function of this variable.

On the basis of expert knowledge concerning the combustion process a set of rules used by the controller was created. Different rules express the state of the process as a set of conditions and the effects that are recommendations for change. RULE 1: IF Tw IS low AND lambda IS rich THEN power IS power_100; $R U L E 2$ : IF Tw IS low AND lambda IS optimal THEN power IS power_60; RULE 3: IF Tw IS low AND lambda IS poor THEN power IS power_20;

\subsection{The Controller for the OFNs}

The controller operation of the directed OFNs requires the use of up and down borders, which are formulated as

$$
\mu_{A}\left(l_{A}\right)=0, \mu_{A}\left(l_{A}^{-}\right)=1, \mu_{A}\left(l_{A}^{+}\right)=1, \mu_{A}\left(p_{A}\right)=0
$$

A graphical interpretation is shown in Fig. 16.10, in which the Ordered Fuzzy Numbers are marked with characteristic border points. Generally it can be assumed that each of the Ordered Fuzzy Numbers can be described by four real numbers:

$$
A=\left(l_{A}, l_{A}^{-}, l_{A}^{+}, p_{A}\right)
$$

In a classic fuzzy controller the resulting membership function is generally a not convex fuzzy set. The use of existing t-norm operators in the inference process will not help to create an OFN according to border points [65]. In [50] there is a proposal which says that instead of t-norm, algebraic operation of multiplication can be used. This is illustrated in Fig. 16.11. A sample controller for OFNs, in particular the inference block and a way to calculate the degree of activation amounts to the

Fig. 16.10 Ordered Fuzzy

Number limit values a positively ordered, $\mathbf{b}$ negatively ordered (a)

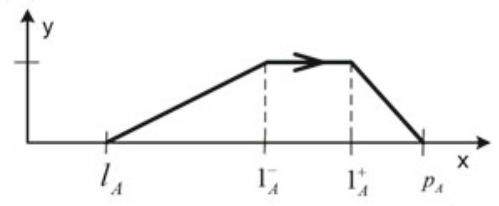

(b)

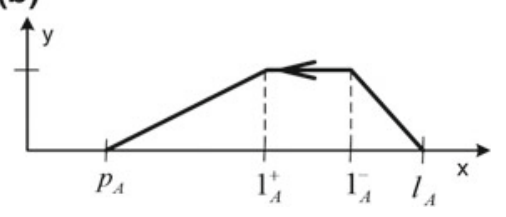




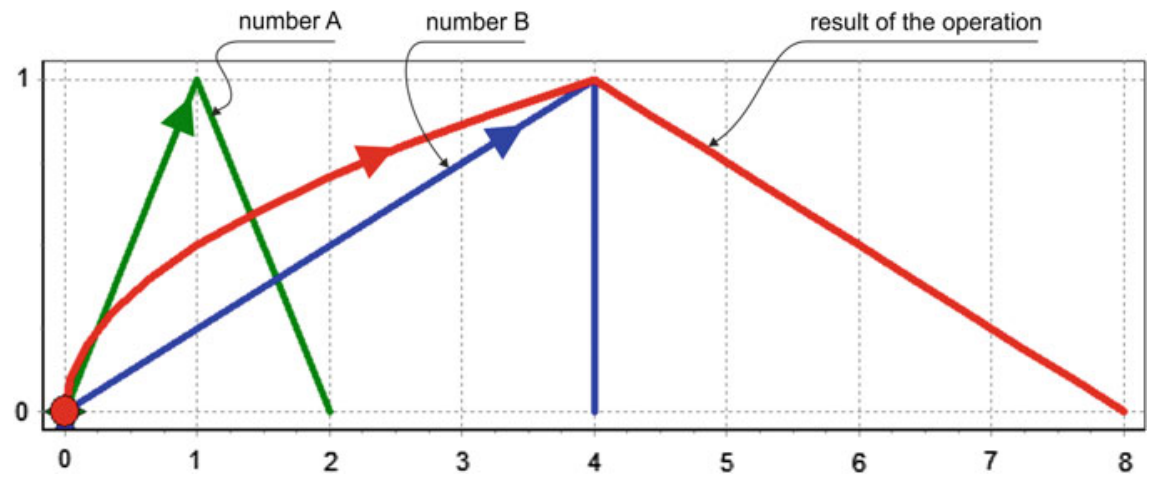

Fig. 16.11 Multiplication of two OFNs

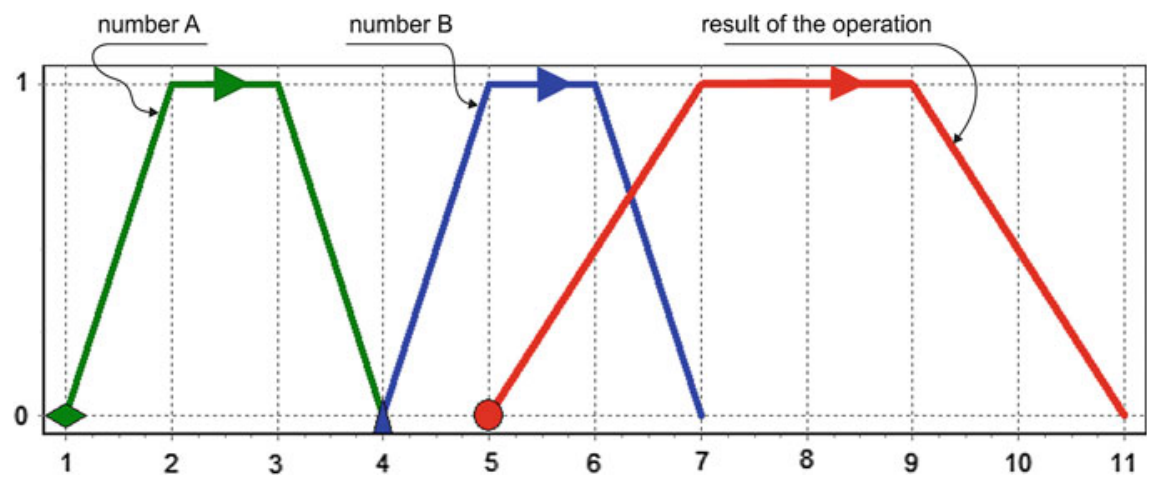

Fig. 16.12 Addition of two OFNs

operation presented above. Having calculated degrees of compliance with each of the rules, an OFN controller can start to cumulate into a single set which is the result. In a classic controller there are s-norm operators. In the controller for OFNs there will be the arithmetic operation of addition. For example, for an ordered number A, $\mathrm{B}$, which is a product of aggregation, addition is shown in Fig. 16.12.

\subsubsection{Directed OFN as a Combustion Trend}

Linking the trend with the directing is associated with reaching the objective of a certain state. In the case when the water temperature of the stove is low, the OFN describing this variable should be positive: Fig. 16.10a, which seeks directing to the boundary point $\mu_{A}\left(p_{A}\right)=0$. Selecting "down" of the OFN emphasizes what value the OFN is going to achieve. For example, the stove water temperature can be a linguistic variable, as shown in Fig. 16.13. A fuzzy number $A$ indicates that the 


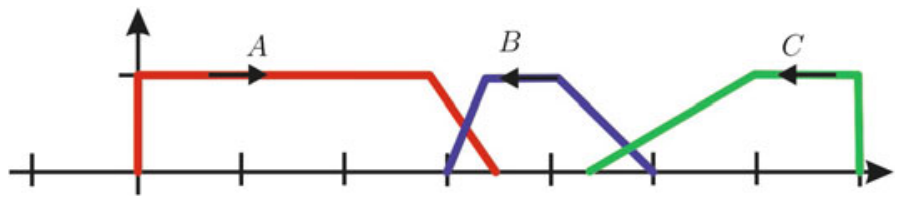

Fig. 16.13 Fuzzy set of input variable 'coil temperature' according to the OFN

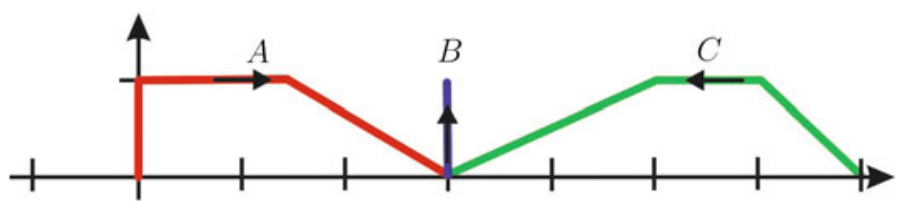

Fig. 16.14 Fuzzy set of input variable $\lambda$ according to the OFN

water is cool but tends to a higher temperature, whereas the number $B$ informs us of the optimum state with a downward trend. The number $C$ is specified for high temperatures, which should not be achieved.

The second variable is the information on the air excess in the exhaust, Fig. 16.14, which is the $\lambda$ ratio. A number says that the mixture is rich and has a small amount of oxidant. In this case the trend as the directing is trying to strive for the $B$ number. Number B describes a mixture where the composition is stoichiometric, that is, everything is burned, causing high efficiency, and the number $C$ contains information about a large amount of oxygen in the exhaust gas and lean fuel mixture. Directing of number $C$ tends to the desired state, which is determined by the $B$ number.

\subsection{Modeling Trend in the Inference Process}

The basis for the construction of a fuzzy controller for OFN, according to the assumption that the number is located within the limits of OFN arms (see Fig. 16.7), is the reconstruction of an inference apparatus in such a way that the resulting product that is the function was the OFN. The proposal of such a construction is shown in this section, where the expert has a method of modeling the direction of the OFN. In the first stage we can say that the output fuzzy set is the CFN convex number. As an example, it is Fig. 16.15 showing the output variable of a blower regulator power. The $D$ number covers the entire range of the blower controller operation. Directing this number at this stage is not established, because this is a convex fuzzy number. In contrast, expert judgment is expressed in the set of rules as a degree of compliance with the rule, that is, a percentage of the convex CFN. For example, evaluation of $+20 \%$ is shown in Fig. 16.16. The number of $D$ was reduced by $20 \%$ on the figure's left side. In contrast, expert judgment $-20 \%$ reduces the vehicle from the right side; this case is shown in Fig. 16.17. For example, having two linguistic variables Tw and $\lambda$, 
Table 16.1 Rules for inference process

\begin{tabular}{l|l|l|l}
\hline \multirow{2}{*}{$T w$} & \multicolumn{3}{l}{ Coefficient $\lambda$} \\
\cline { 2 - 4 } & Rich $(\%)$ & Optimal (\%) & Poor (\%) \\
\hline Low & +60 & +30 & -20 \\
\hline Average & +50 & +20 & -10 \\
\hline High & +30 & +50 & -5 \\
\hline
\end{tabular}

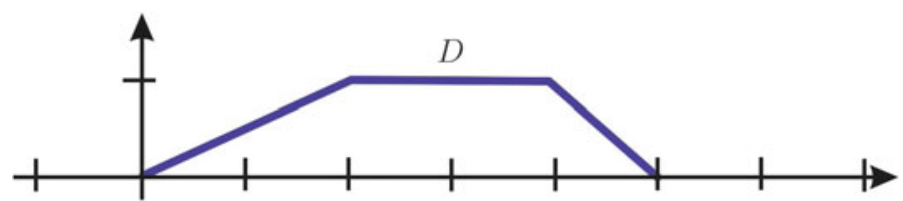

Fig. 16.15 Fuzzy set of output variable 'power' according to CFN

Fig. 16.16 CFN number for the expert proposal $+20 \%$

Fig. 16.17 CFN number for the expert proposal $-20 \%$
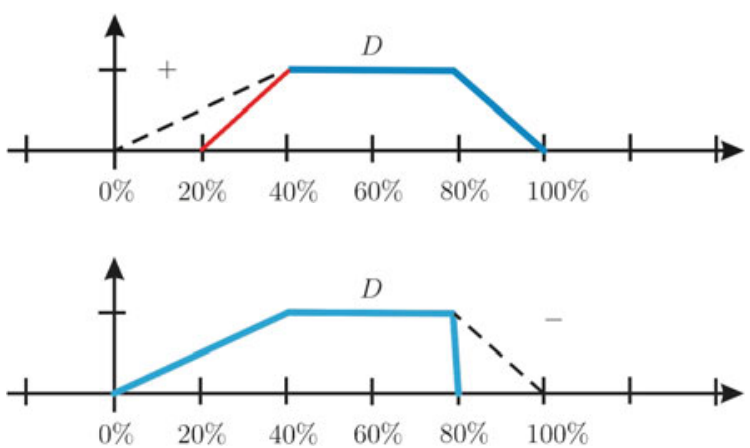

taking into account expert judgment expressed as the degree of compliance with the percentage share of the output number, a set of rules will form as in Table 16.1. Signs in front of the percentages can be interpreted as the imposed direction to CFN from Fig. 16.15. In other words, modeling the trend in the inference process is reduced to the use of OFNs in the form of CFNs as shown in Fig. 16.15. The result of running the rules is imposed orientation: positive when we have a plus, and negative when we have a minus. Accordingly, for Fig. 16.15 it will be Fig. 16.18, For Fig. 16.17, we have directing presented as in Fig. 16.19. The added value of this solution is the ability to regulate the OFN direction at the inference stage. The percentages given by an expert can be regarded as a temporary modification. Directing of OFNs will be established when we accumulate all the rules. The established resulting OFN will allow us to go into the process of defuzzification. Another feature of this solution is the ability to reduce the surface area of the OFN. This feature can be particularly important from the perspective of defuzzification. Using the method of defuzzification associated with the surface area narrows the result already at the inference stage. 
Fig. 16.18 Example OFN, the result of the $+20 \%$ rule
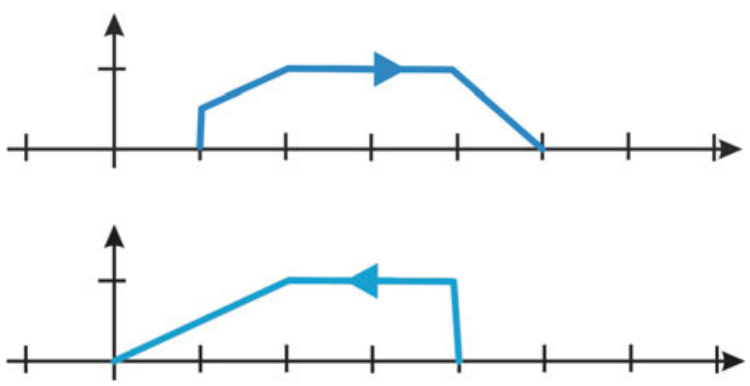

Fig. 16.19 Example OFN, the result of the $-20 \%$ rule

\subsection{Conclusions}

Handling of the Ordered Fuzzy Number in engineering applications is an alternative to convex fuzzy numbers. Meeting this task entails building an ordered fuzzy controller, which uses directing. In the context of this chapter 'ordered' expresses the trend of the studied phenomenon. And trend describes the tendency of a given variable. With the construction of a classic controller the authors introduced a variable associated with the increase in temperature per unit of time. The observed increase would allow determination of the short-term changes in water temperature. The use of OFN enabled following the trend in every other variable describing the combustion process. Returning to the main thread of the work, the aim of which was to use the trend in the combustion process, the authors focused on the inference process. The ability to model the directing of the OFN, which can be seen in the penultimate section, expands the possibilities of using the trend. Applying this approach will emphasize the trend in the inference process. By manipulating the percentage value of the medium of the output OFN, we received a modification of the resulting function. The use of this manipulation can be helpful when we want to change directing or reduce the OFN medium. Reducing the medium of a number, that is, fuzzification process input information, and applying methods such as MCOG, MF, or GR [15] sensitive to directing contribute to a better reflection of the process trend.

\section{References}

1. Apiecionek, L., Czerniak, J.M.: QoS solution for network resource protection. In: Informatics 2013: Proceedings of the Twelfth International Conference on Informatics. pp. 73-76 (2013)

2. Apiecionek, L., Romantowski, M.: Secure IP network model. Comput. Method Sci. Technol. 19(4), 209-213 (2013)

3. Apiecionek, L., Czerniak, J.M., Zarzycki, H.: Protection tool for distributed denial of services attack. In: Beyond Databases, Architectures and Structures, BDAS, pp. 405-414. Springer, Berlin (2014)

4. Apiecionek, L., Czerniak, J.M., Dobrosielski, W.T.: Quality of services method as a DDoS protection tool. In: Intelligent Systems' 2014, Vol 2: Tools, Architectures, Systems, Applications vol. 323, pp. 225-234 (2015) 
5. Bednarek, T., Kosiński, W., Wȩgrzyn-Wolska, K.: On orientation sensitive defuzzification functionals. Artificial Intelligence and Soft Computing, pp. 653-664. Springer, Berlin (2014)

6. Chwastyk, A., Kosiński, W.: Fuzzy calculus with applications. Math. Appl. 41(1), 47-96 (2013)

7. Czerniak, J.M., Ewald, D.: A new mglaber approach as an example of novel artificial acari optimization. 545-557 (2016). doi:10.1007/978-3-319-34099-9_42

8. Czerniak, J., Dobrosielski, W., Apiecionek, L.: Representation of a trend in OFN during fuzzy observance of the water level from the crisis control center. In: Proceedings of the Federated Conference on Computer Science and Information Systems, IEEE Digital Library, ACSIS 5, 443-447 (2015)

9. Czerniak, J.M., Zarzycki, H., Ewald, D.: AAO as a new strategy in modeling and simulation of constructional problems optimization. Simulation Modelling Practice and Theory, vol. 76C, pp. 22-33. Elsevier (2017). http://doi.org/10.1016/j.jocs.2017.05.028

10. Czerniak, J.M., Dobrosielski, W., Zarzycki, H., Apiecionek, L.: A proposal of the new owlant method for determining the distance between terms in ontology. IntelligenT Systems' 2014, vol 2: Tools, Architectures, Systems, Applications, vol. 323 pp. 235-246 (2015)

11. Czerniak, J., Smigielski, G., Ewald, D., Paprzycki, M.: New proposed implementation of ABC method to optimization of water capsule flight. In: Proceedings of the Federated Conference on Computer Science and Information Systems, IEEE Digital Library, ACSIS 5 pp. 489-493 (2015)

12. Czerniak, J., Apiecionek, Ł., Zarzycki, H., Ewald, D.: Proposed caeva simulation method for evacuation of people from a buildings on fire. Adv. Intell. Syst. Comput 401, 315-326 (2016)

13. Czerniak, J.M., Zarzycki, H.: Artificial acari optimization as a new strategy for global optimization of multimodal functions. J. Comput. Sci. Elsevier (2017). http://doi.org/10.1016/j. jocs.2017.05.028

14. Czerniak, J.M., Dobrosielski, W.T., Apiecionek, Ł., Ewald, D., Paprzycki, M.: Practical Application of OFN Arithmetics in a Crisis Control Center Monitoring, pp. 51-64. Springer International Publishing, Cham (2016). doi:10.1007/978-3-319-40132-4_4

15. Dobrosielski, W.T., Szczepanski, J., Zarzycki, H.: A proposal for a method of defuzzification based on the golden ratio - gr. Novel Developments in Uncertainty Representation and Processing, pp. 75-84. Springer International Publishing, Berlin (2016)

16. Dubois, D., Prade, H.: Operations on fuzzy numbers. Int. J. Syst. Sci. 9(6), 613-626 (1978)

17. Dyczkowski, K.: A less cumulative algorithm of mining linguistic browsing patterns in the world wide web (2007)

18. Dyczkowski, K., Wygralak, M.: On triangular norm-based generalized cardinals and singular fuzzy sets. Fuzzy Sets Syst. 133(2), 211-226 (2003)

19. Ewald, D., Czerniak, J.M., Zarzycki, H.: Approach to solve a criteria problem of the ABC algorithm used to the wbdp multicriteria optimization. Intelligent Systems' 2014, vol 1: Mathematical Foundations, Theory, Analyses 322, 129-137 (2015)

20. Filev, D.P., Yager, R.R.: A generalized defuzzification method via bad distributions. Int. J. Intell. Syst. 6(7), 687-697 (1991)

21. Kacprzak, D.: Income and total cost of a firm expressed by ordered fuzzy numbers (in polish)(przychód i koszt całkowity przedsiebiorstwa wyrażony przy użyciu skierowanych liczb rozmytych), zarzaadzanie i finanse. J. Manag. Financ. 2(1) (2012)

22. Kacprzak, D., Kosiński, W.: Optimizing firm inventory costs as a fuzzy problem. Stud. Log. Gramm. Rhetor. 37, 89-105 (2014)

23. Kacprzak, D., Kosiński, W., Prokopowicz, P.: Fuzziness - representation of dynamic changes by ordered fuzzy numbers. Stud. Fuzziness Soft Comput. 243, 485-508 (2009)

24. Kacprzak, D., Kosiński, W., Kosiński, W.K.: Financial stock data and ordered fuzzy numbers. In: Artificial Intelligence and Soft Computing : 12th International Conference, ICAISC'2013. pp. 259-270. IEEE (2013)

25. Kacprzak, M., Kosiński, W.: On lattice structure and implications on ordered fuzzy numbers. In: Proceedings of EUSFLAT. Artificial Intelligence and Soft Computing, vol. 7267 of LNCS, pp. 247-255 (2011) 
26. Kacprzak, M., Starosta, B., Wȩgrzyn-Wolska, K.: Metasets and opinion mining in new decision support system. In: Rutkowski, L., Korytkowski, M., Scherer, R., Tadeusiewicz, R., Zadeh, L.A., Zurada, J.M. (eds.) Artificial Intelligence and Soft Computing, Part II. Lecture Notes in Artificial Intelligence, vol. 9120, pp. 625-636. Springer International Publishing, Berlin (2015)

27. Kacprzak, M., Starosta, B., Wȩgrzyn-Wolska, K.: New approach to decision making. In: Abraham, A., Wegrzyn-Wolska, K., Hassanien, A.E., Snasel, V., Alimi, A.M. (eds.) In: Proceedings of the Second International Afro-European Conference for Industrial Advancement AECIA 2015. Advances in Intelligent Systems and Computing, vol. 427, pp. 397-407. Springer International Publishing, Berlin (2015)

28. Kacprzyk, J., Yager, R.R.: Emergency-oriented expert systems: a fuzzy approach. Inf. Sci. 37(1), 143-155 (1985)

29. Kacprzyk, J.: Fuzzy Sets in System Analysis (in Polish). PWN (1986)

30. Kocaarslan, I., Çam, E., Tiryaki, H.: A fuzzy logic controller application for thermal power plants. Energy convers. manag. 47(4), 442-458 (2006)

31. Kosinski, W., Prokopowicz, P.: Fuzziness - Representation of dynamic changes ? In: Stepnicka, $\mathrm{M}$ and Novak, V and Bodenhofer, U (ed.) New Dimensions in Fuzzy Logic and Related Technologies, Proceedings. Vol I, pp. 449-456. European Soc Fuzzy Log and Technol, Univ Ostrava, Ostravska Univ \& Ostrave, Dvorakova 7, Ostrava 1, 701 03, Czech Republic (2007), 5th Conference of the European-Society-for-Fuzzy-Logic-and-Technology, Ostrava, Czech Republic, 11-14 Sep 2007

32. Kosinski, W., Chwastyk, A.: Ordered fuzzy numbers in financial stock and accounting problems. In: Proceedings of the 2013 Joint IFSA World Congress and Nafips Annual Meeting (IFSA/NAFIPS), pp. 546-551 (2013)

33. Kosiński, W., Prokopowicz, P., Ślęzak, D.: On Algebraic Operations on Fuzzy Reals. In: Rutkowski L and Kacprzyk J (eds.) Neural Networks and Soft Computing: Proceedings of the Sixth International Conference on Neural Networks and Soft Computing, Zakopane, Poland, 11-15 June 2002, pp. 54-61. Physica-Verlag HD, Heidelberg (2003). doi:10.1007/978-3-79081902-1_8

34. Kosiński, W., Prokopowicz, P., Ślęzak, D.: Ordered fuzzy numbers. Bull. Pol. Acad. Sci. Ser. Sci. Math 51(3), 327-338 (2003)

35. Kosinski, W., Prokopowicz, P., Slezak, D.: Calculus with fuzzy numbers. In: Bolc, L and Michalewicz, $\mathrm{Z}$ and Nishida, $\mathrm{T}$ (ed.) Intelligent Media Technology For Communicative Intelligence. Lecture Notes in Artificial Intelligence, vol. 3490, pp. 21-28 (2004), 2nd Intenational Workshop on Intelligent Media Technology for Communicative Intelligence, Warsaw, Poland, 13-14 Sep 2004

36. Kosiński, W., Prokopowicz, P., Rosa, A.: Defuzzification functionals of ordered fuzzy numbers. IEEE Trans. Fuzzy Syst. 21(6), 1163-1169 (Dec 2013). doi:10.1109/TFUZZ.2013.2243456

37. Kowalewicz, A.: Podstawy procesów spalania. Wydawnictwa Naukowo-Techniczne (2000)

38. Lebiediewa, S., Zarzycki, H., Dobrosielski, W.: A new approach to the equivalence of relational and object-oriented databases. In: Novel Developments in Uncertainty Representation and Processing, pp. 85-93. Springer International Publishing, Berlin (2016)

39. Łukasiewicz, J.: Elements of Mathematical Logic, vol. 31. Macmillan, New York (1963)

40. Mahdiani, H., Banaiyan, A., Javadi, M.H.S., Fakhraie, S., Lucas, C.: Defuzzification block: new algorithms, and efficient hardware and software implementation issues. Eng. Appl. Artif. Intell. 26(1), 162-172 (2013). http://www.sciencedirect.com/science/article/pii/ S0952197612001601

41. Marszalek, A., Burczyński, T.: Financial fuzzy time series models based on ordered fuzzy numbers. In: Pedrycz, W., Chen, S.M. (eds.) Time Series Analysis, Modeling and Applications: A Computational Intelligence Perspective, pp. 77-95. Springer, Berlin (2013)

42. Marszalek, A., Burczyński, T.: Modelling financial high frequency data using ordered fuzzy numbers. In: Rutkowski, L., Korytkowski, M., Scherer, R., Tadeusiewicz, R., Zadeh, L.A., Zurada, J.M. (eds.) Artificial Intelligence and Soft Computing: 12th International Conference, ICAISC 2013, Zakopane, Poland, 9-13 June 2013, Proceedings, Part I, pp. 345-352. Springer, Berlin (2013) 
43. Marszalek, A., Burczyński, T.: Modeling and forecasting financial time series with ordered fuzzy candlesticks. Inf. Sci. 273, 144-155 (2014). http://www.sciencedirect.com/science/ article/pii/S0020025514003107

44. Mikolajewska, E., Mikolajewski, D.: Wheelchair development from perspective of physical therapists and biomedical engineers. Adv. Clin. Exp. Med. 19(6), 771-776 (2010)

45. Mikolajewski, D., Mikolajewska, E.: Exoskeletons in neurological diseases - current and potential future applications. Adv. Clin. Exp. Med. 20(2), 227-233 (2011)

46. Mikolajewska, E., Mikolajewski, D.: Neuroprostheses for increasing disabled patients' mobility and control. Adv. Clin. Exp. Med. 21(2), 263-272 (2012)

47. Mikolajewska, E., Mikolajewski, D.: Ethical considerations in wider use of brain-computer interfaces in patients with neurological deficits. Cent. Eur. J. Med. 8(6), 720-724 (2013)

48. Mikolajewska, E., Mikolajewski, D.: Integrated it environment of disabled people - a new concept. Cent. Eur. J. Med. 9(1), 177-182 (2014)

49. Mikolajewska, E., Mikolajewski, D.: The prospects of brain-computer application in children. Cent. Eur. J. Med. 9(1), 74-79 (2014)

50. Prokopowicz, P.: Algorithmization of operations on fuzzy numbers and its applications (in polish: Algorytmizacja działań na liczbach rozmytych i jej zastosowania)

51. Prokopowicz, P.: Methods based on ordered fuzzy numbers used in fuzzy control. In: Proceedings of the Fifth International Workshop on Robot Motion and Control, 2005. RoMoCo '05. pp. 349-354 (2005). doi:10.1109/ROMOCO.2005.201448

52. Prokopowicz, P.: Adaptation of rules in the fuzzy control system using the arithmetic of ordered fuzzy numbers. In: Rutkowski, L., Tadeusiewicz, R., Zadeh, L., Zurada, J. (eds.) Artificial Intelligence and Soft Computing - ICAISC 2008, Lecture Notes in Computer Science, vol. 5097, pp. 306-316. Springer, Berlin (2008). doi:10.1007/978-3-540-69731-2_30

53. Prokopowicz, P.: Flexible and simple methods of calculations on fuzzy numbers with the ordered fuzzy numbers model. In: Rutkowski, L., Korytkowski, M., Scherer, R., Tadeusiewicz, R., Zadeh, L., Zurada, J. (eds.) Artificial Intelligence and Soft Computing, Lecture Notes in Computer Science, vol. 7894, pp. 365-375. Springer, Berlin (2013). doi:10.1007/978-3-64238658-9_33

54. Prokopowicz, P.: Analysis of the changes in processes using the Kosinski's fuzzy numbers. In: Ganzha, M., Maciaszek, L., Paprzycki, M. (eds.) Proceedings of the 2016 Federated Conference on Computer Science and Information Systems. Annals of Computer Science and Information Systems, vol. 8, pp. 121-128. IEEE (2016). doi:10.15439/2016F140

55. Prokopowicz, P.: Proceedings of the Second International Afro-European Conference for Industrial Advancement AECIA 2015, chap. The Directed Inference for the Kosinski's Fuzzy Number Model, pp. 493-503. Springer International Publishing, Cham (2016). doi:10.1007/978-3319-29504-6_46

56. Ruusunen, M., Leiviskä, K.: Fuzzy modelling of carbon dioxide in a burning process. Control Eng. Pract. 12(5), 607-614 (2004)

57. Smigielski, G., Dygdała, R., Zarzycki, H., Lewandowski, D.: Real-time system of delivering water-capsule for firefighting. In: Advances in Intelligent Systems and Computing. vol. 534, pp. 102-111. Springer International Publishing, Berlin (2016)

58. Sobol, I., Kacprzak, D., Kosiński, W.: Optimizing of a company's cost under fuzzy data and optimal orders under dynamic conditions. Optimum. Studia Ekonomiczne 5, 172-187 (2014)

59. Sovacool, B.K., Cooper, C.: Nuclear nonsense: why nuclear power is no answer to climate change and the world's post-kyoto energy challenges. Wm. and Mary Envtl. L. and Pol'y Rev. 33, 1 (2008)

60. Stachowiak, A., Dyczkowski, K.: A similarity measure with uncertainty for incompletely known fuzzy sets. In: Proceedings of the 2013 Joint IFSA World Congress and NAFIPS Annual Meeting (IFSA/NAFIPS), pp. 390-394 (2013)

61. Stachowiak, A., Dyczkowski, K., Wojtowicz, A., Zywica, P., Wygralak, M.: A bipolar view on medical diagnosis in ovaexpert system (2016)

62. Sugeno, M.: An introductory survey of fuzzy control. Inf. Sci. 36, 59-83 (1985). http://www. sciencedirect.com/science/article/pii/002002558590026X 
63. Szczeniowski, B.: Obliczanie wykresów składu spalin. Przegląd Techniczny 39-40, 573-577 (1931)

64. Yager, R.R., Filev, D.P.: Essentials of Fuzzy Modeling and Control. New York (1994)

65. Zadeh, L.: Fuzzy sets. Inf. Control 8(3), 338-353 (1965). http://www.sciencedirect.com/ science/article/pii/S001999586590241X

66. Zadeh, L.A.: Toward a theory of fuzzy systems. Fuzzy sets, fuzzy logic, and fuzzy systems: selected papers by Lotfi A. Zadeh (1996)

67. Zadeh, L.A.: Is there a need for fuzzy logic? Inf. Sci. 178(13), 2751-2779 (2008)

68. Zarzycki, H., Czerniak, J., Lakomski, D., Kardasz, P.: Performance comparison of crm systems dedicated to reporting failures to it department. In: Software Engineering: Challenges and Solutions, Advances in Intelligent Systems and Computing, vol. 504, pp. 133-146. Springer International Publishing, Berlin (2016)

Open Access This chapter is licensed under the terms of the Creative Commons Attribution 4.0 International License (http://creativecommons.org/licenses/by/4.0/), which permits use, sharing, adaptation, distribution and reproduction in any medium or format, as long as you give appropriate credit to the original author(s) and the source, provide a link to the Creative Commons license and indicate if changes were made.

The images or other third party material in this chapter are included in the chapter's Creative Commons license, unless indicated otherwise in a credit line to the material. If material is not included in the chapter's Creative Commons license and your intended use is not permitted by statutory regulation or exceeds the permitted use, you will need to obtain permission directly from the copyright holder.

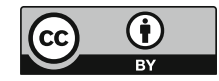

